\title{
El Centro de Documentación arqueoastronómica y afines
}

César Israel Rodriguez Carías Santos Vito Véliz Bertilio Amaya

\section{Resumen}

El Departamento de Arqueoastronomía, busca enriquecer y organizar su acervo bibliográfico para poder desempeñar sus labores con más solvencia. El esfuerzo ha sido obtener por todos los medios posibles libros, revistas, artículos, videos y sitios web con información sobre arqueoastronomia y ciencias relacionadas.

El objetivo es recopilar bibliografía sobre arqueoastronomia y campos afines para el mejor desempeño de nuestras labores de investigación y de enseñanza y para colaborar con otros investigadores y con alumnos. Se inicio con un inventario del material bibliográfico con que dispone el departamento de Arqueoastronomia, para así evaluar las necesidades y fortalezas inmediatas para la formación del Centro de Documentación. Se ha acondicionado un área de trabajo y se ha colocado la literatura existente previa organización. Se ha contactado a particulares e instituciones para lograr donaciones y/o canjes, Se buscara presupuesto para comprar libros o suscripciones de revistas, se elaboran fichas para todas las referencias, se ha obtenido material digital y se ha colocado en carpetas, se hace revisión de sitios de internet científicos, se obtienen videos para presentaciones y se revisan centros de documentación institucionales.

Se busca disponer de un centro de documentación único en su género, organizado y accesible, para profesores y estudiantes, que permita desarrollar convenientemente la docencia e investigación en el departamento de Arqueoastronomia.

Podemos concluir que la carencia de material bibliográfico idóneo dificulta la enseñanza aprendizaje. La existencia de material bibliográfico actualizado promueve una mejora en la educación, investigación y desarrollo cultural. Algo que buscamos apoyar con el Centro de Documentación.

Palabras clave: Arqueoastronomia, Centro de Documentación, Astronomía Cultural. 


\section{Abstract}

The Department of Archeoastronomy, seeks to enrich and organize their bibliographic order to perform their duties with more solvency acquis. The effort has been obtained by all means possible books, magazines, videos and websites with information on archaeoastronomy and related sciences.

The objective is to collect literature on archaeoastronomy and related to the better performance of our research and teaching fields and to collaborate with other researchers and students. She began with an inventory of bibliographic material available Archaeoastronomy department, in order to assess immediate needs and strengths to the formation of the Documentation Centre. Conditioning has a work area and has placed upon existing literature organization. It has contacted individuals and institutions for grants and / or exchanges, budget is sought to buy books or magazine subscriptions, tabs for all referrals are made, was obtained digital material and placed in folders, revision is made scientists internet sites, videos for presentations are obtained and institutional documentation centers are reviewed.

It seeks to have a documentation center unique in its kind, organized and accessible to teachers and students, allowing a proper developing teaching and research in the department of Archaeoastronomy. We conclude that the lack of suitable bibliographic material hinders the learning. The existence of updated bibliography promotes improved education, research and cultural development. Something that we seek to support the Documentation Centre.Keywords: Solar eclipses, lunar eclipses, Copan.

Keywords: Archaeoastronomy, Documentation Centre, Cultural Astronomy.

César Israel Rodriguez Carías, Santos Vito Véliz, Bertilio Amaya, Departamento de Arqueoastronomía y Astronomía Cultural, Universidad Nacional Autónoma de Honduras 
Introducción

Desde épocas pasadas el ser humano se ha preocupado por entender el significado de los movimientos aparentes del sol y cuerpos celestes, las culturas antiguas como la maya, dejaron evidencia de sus observaciones astronómicas en numerosos monumentos, altares, estelas y códices. Entre los objetivos de la Arqueoastronomía está comprobar que nuestros antepasados tuvieron razones para ubicar y orientar sus obras arquitectónicas, conocer la relación de monumentos entre sí y con los astros y también la relación entre sitios arqueológicos con esos mismos astros.

La Arqueoastronomía como ciencia estudia la relación de las culturas antiguas con el cosmos, Se considera que los orígenes de la disciplina se remontan al trabajo de Heinrich Nissen, arquitecto alemán de mediados del siglo XIX, que participo en varias expediciones arqueológicas y fue el primero que propuso que los antiguos griegos y romanos orientaron sus templos astronómicamente. Su trabajo fue seguido por Sir Norman Lockyer, el editor de la revista Nature; que publico el clásico The dawn of astronomy. (1894). Posteriormente, en 1901, Lockyer haría una datación astronómica de Stonehenge que colocaría la construcción del monumento entre el 1600 y el 2000 B.C. En 1906 publicaría Stonehenge and Other British Monuments Astronomically Considered. El astrónomo Gerald Hawkins, quien fue el primero en utilizar computadoras para determinar los alineamientos astronómicos de Stonehenge. Hawkins encontró alineaciones a los solsticios y las paradas de la luna.

A finales de 1960 el arqueólogo escocés Euan McKie, propuso usar la palabra "arqueoastronomía" para denotar este nuevo campo científico. En los 70 fue Edwin Krupp, el director del observatorio Griffith, quien retomo un término de Lockyer para definir la disciplina como Arqueoastronomía, denotando la investigación de las antiguas astronomías (Arqueoastronomia.org).

Es precisamente en los años 70 cuando la arqueoastronomía alcanza el status de disciplina científica gracias al trabajo de Alexander Thom, profesor de ingeniería de la universidad de Oxford, quien con la publicación de su trabajo Megalithic sites in Britain (1967), sentó las bases metodológicas de la arqueoastronomía durante las últimas décadas del siglo XX. Alexander Thom inspecciono cientos de lugares megalíticos en Gran Bretaña. En 1977 John B. Carlson, director del Centro de Arqueoastronomía de la Universidad de Maryland, edita la revista Archaeoastronomy y en 1979 aparece la revista Archaeoastronomy, por el Prof. Michael Hoskin, como suplemento de Journal for the History of Astronomy. En 1992 recibe apoyo al surgir la European Society for Astronomy in Culture (SEAC). 
Según Cerdeño y Rodríguez, en (Complutum, 2009), la arqueoastronomía no es una rama de la Astronomía sino de la Arqueología, y solo se justifica si puede proporcionar respuestas a interrogantes planteados desde la Arqueología o Antropología. Ejemplo trabajos de Iwaniszewski en 2009, Pasztor en 2009 y Belmonte 0 Esteban también en 2009.

Se considera la Arqueoastronomía como la relación de las sociedades antiguas con el Cosmos.

Pasztor considera que la Arqueoastronomía es una subdisciplina de la Arqueología y por ello las precisiones de los Arqueólogos pueden mejorar y complementar el punto de vista de los astrónomos. Se señala que los investigadores deben de ser los supervisores de su difusión y divulgación.

Es notoria la faceta cultural de observación del cielo, que practicaron todas las sociedades de una u otra manera. El cielo fue y es un referente universal, un patrimonio común de todas las sociedades presentes y pasadas. se debe crear una base de datos de todos los lugares estudiados desde un punto de vista Arqueoastronómico, con la finalidad de identificarlos, estudiarlos y protegerlos.

Como disciplina la Arqueoastronomía según Belmonte, es una materia auxiliar de las Ciencias Sociales como: la Arqueología, la Antropología o la Historia, y en que el empirismo y la metodología de una ciencia experimental, como la Astronomía es determinante. La Arqueoastronomía no es una línea de investigación dentro de la astrofísica moderna, ni sirve a su fin fundamental cual es el avance del conocimiento físico del universo. Es una especialidad que se enmarca en los estudios antropológicos, al servicio de disciplinas como la Arqueología (Lull, 2006).

Se puede catalogar a la Arqueoastronomia en cualquier estudio de las prácticas de observación del cielo con fines culturales (religión, adivinación, arquitectura, decoración, pintura, planificación de ciudades, medida del tiempo, navegación, etc.). Otro concepto de Arqueoastronomia es que intenta averiguar el papel que jugó el cielo en las manifestaciones culturales de los grupos de épocas pasadas.

Existe preocupación por la escasa presencia de la Arqueoastronomia o la Astronomía Cultural en universidades. En España su presencia se da en facultades de astronomía y física, mientras en humanidades es prácticamente inexistente.

- Nos encontramos con cuestiones tan variadas como: "calendarios, observación práctica, cultos y mitos, representación simbólica de eventos, conceptos y 
objetos astronómicos, orientación astronómica de tumbas, templos, santuarios y centros urbanos, cosmología tradicional y la aplicación ceremonial de tradiciones astronómicas" (E. Krupp, en Las leyes del cielo, de J. A. Belmonte.), todas ellas unificadas por un tratamiento que las ubica dentro de su contexto cultural, social, económico e histórico

- Por sus características, es ésta un área que requiere indefectiblemente del trabajo interdisciplinario de astrónomos, antropólogos, arqueólogos, historiadores, arquitectos, sociólogos, etc. Lo cual constituye obviamente un enorme desafío, ya que implica la creación de un lenguaje y pautas de trabajo comunes entre representantes de disciplinas generalmente muy alejadas entre sí.

La Arqueoastronomía no solo trata de observar eventos celestes, de descubrir orientaciones o de tomar medidas de monumentos antiguos, sino de aproximarnos a la visión que de todo ello tenían los grupos humanos que los construyeron.

La Antropología juega un papel importante en el desarrollo de la Arqueoastronomía, pues fue la curiosidad del hombre lo que lo llevo a levantar su Mirada y tratar de comprender lo que miraba, porque el sol aparecía y desaparecía, como aparecía la luna y las estrellas etc.

Los estudios de Arqueoastronomía ya sea desde el campo de la Arqueología o de la Astronomía deben cumplir dos aspectos fundamentales:

- El rigor en los métodos y técnicas empleados.

- El debate conceptual para una base sólida.

El enfoque cultural de la arqueoastronomía y la etnoastronomía y su fundamento multidisciplinario la convierte en una herramienta para entender las sociedades humanas y la profunda relación mutua entre la astronomía y la cultura.

En la investigación arqueoastronómica se combina el conocimiento de ciencias humanas (la antropología, la arqueología, etnohistoria, y la historia), con métodos cuantitativos, especialmente la astronomía de posición, la topografía y la estadística. 
El arqueoastrónomo usa herramientas como los teodolitos, estaciones totales, brújulas magnéticas, GPS y programas de simulación astronómica virtual así como de SIG (sistemas de información geográfica). La metodología del arqueoastrónomo generalmente pasa por tres etapas:

- Trabajo previo. Comienza escogiendo un lugar, una cultura o un conjunto de restos o evidencia arqueológica para su posterior estudio. Tras ello se debe realizar una revisión exhaustiva de la literatura antropológica, arqueológica e histórica relacionada con el estudio. Tras ello se planifica el trabajo de campo y los objetivos. Una actividad importante es la revisión de mapas y planos del sitio (si es que los hay) ya que examinar un plano indica la complejidad del lugar y su posible uso para la realización de observaciones astronómicas (Arqueoastronomia.org).

- Trabajo de campo. Una vez en el sitio de interés se procede a hacer un reconocimiento en busca de las evidencias de estructuras o vestigios utilizados para la observación astronómica o actividades relacionadas. Generalmente se realizan observaciones del sol, la luna y otros astros de interés, en momentos específicos y de importancia como pueden ser los solsticios, el equinoccio 0 los lunasticios. Igualmente se procede a la medición de orientaciones y alineamientos presentes en las estructuras estudiadas.

Lo más importante al momento de realizar mediciones es lograr establecer, de la forma más precisa posible. El Norte verdadero (geográfico, astronómico) del lugar, para tener una referencia correcta en las mediciones de orientaciones astronómicas.

- Análisis y síntesis de los datos recogidos. Una vez recopilados los datos de investigación de campo se procede a reducir los datos y compararlos con las hipótesis planteadas y contrastarlos con la evidencia cultural previamente estudiada. En el proceso de análisis de los datos se utilizan herramientas informáticas como el software de simulación astronómica (o planetarios) y el software de proceso de datos topográficos o geográficos (o SIG). Por medio del primero se simulan los cielos para una época determinada, teniendo en cuenta variables importantes como la precesión o la refracción; y por medio del segundo se analizan en conjunto los datos geográficos y topográficos, se realizan planos y mapas y se miden azimuts, altitudes y otros (Arqueoastronomia.org). 
Como puede notarse para los estudios de Arqueoastronomía es muy importante una base bibliográfica científica digital e impresa, que nos ayude a desarrollar las investigaciones en tiempo y forma.

Algunos trabajos de Arqueoastronomía argentina son:

Meteoritos de Campo del Cielo: Impactos en la cultura aborigen, Astronomía Aborigen del Chaco: Mocovíes I. El Sol y la Luna como hitos del tiempo y el espacio entre los mocovíes del Chaco argentino. (Arqueoastronomia.org/artículos).

Para trabajos de esta magnitud es indispensable la existencia de una buena cantidad de material bibliográfico y el apoyo de organizaciones internacionales como:

- Sociedad Interamericana de Astronomía en la Cultura (SIAC)

- European Society of Astronomy in Culture (SEAC)

- The International Society for Archaeoastronomy and Astronomy in Culture (ISAAC)

- Seminario de Arqueoastronomía Mexicana (ENAH)

- Conferencias Oxford

- Journal for the History of Astronomy

- Conferences on Astronomy and Cultural Diversity

- Archaeoastronomy Journal

- Journal of Skyscape Archaeology

- Arqueoastronomia.org. Sitio dedicado a la difusión de la Arqueoastronomía en Hispanoamérica.

Estas organizaciones podrían ayudar al desarrollo de la Arqueoastronomía en Honduras sobre todo accediendo a su base de datos de investigaciones y bibliografía.

En el caso de Honduras las observaciones en las ruinas de Copan, sus altares, sus edificios, sus plazas y estelas como la B y el Altar $Q$ tienen un significado arqueoastronómico, ya que presentan un alineamiento o nos cuentan sobre 
eventos de observaciones a simple vista pasadas. También los Mayas productos de estas observaciones nos heredaron el calendario más preciso hasta nuestros días (Chinchilla, 1982).

Dado que esta ciencia es relativamente nueva y que Honduras es pionera en su enseñanza por medio de la Asignatura "Introducción a la Arqueoastronomía", es necesaria la obtención de material bibliográfico de apoyo para el desarrollo de la clase, esta bibliografía comprende literatura de Astronomía, Antropología, Arqueología entre otras ciencias complementarias.

La bibliografía de interés incluye autores que han realizado importantes publicaciones acerca de investigaciones arqueológicas en Honduras como: Claude Baudez, (Baudez, Claude. 1966. Niveaux céramiques au Honduras: une reconsidération de l'évolution culturelle), Pineda Portillo (Pineda, Noé.1997 Geografía de Honduras). Doris Stone (Stone, Doris. 1957 The archaeology of central and southern Honduras) y Vito Véliz (Véliz, Vito. 1988 Rudimentos de Antropología) y (1983, Síntesis histórica de la arqueología en Honduras).

Así como importantes autores extranjeros pioneros en la investigación arqueoastronómica como: Juan Antonio Belmonte, José Lull, Jesús Galindo Trejo y Anthony Aveni entre otros.

La obtención de material bibliográfico actualizado sumado al ya existente en el Departamento, nos permitirá la creación de un Centro de documentación arqueoastronómica, de utilidad a docentes y alumnos, brindándonos la capacidad de orientar a las nuevas generaciones a un nuevo campo de investigación científica.

Es importante en nuestro país invertir los recursos en la educación e investigación, Valores como la lectura son importantes para rescatar nuestra sociedad, la existencia de Centros de Documentación permite poder acceder como docente y alumno a conocer más acerca de un tema de interés, desarrollar investigaciones bibliográficas y de ayuda al trabajo de campo. Favorecen el desarrollo de la docencia y la formación intelectual del educando. Todo esto favorece la calidad de educación brindada por la UNAH y la sitúa en los peldaños de excelencia global.

\section{Objetivo}

Recopilar bibliografía sobre arqueoastronomía y campos afines para el mejor desempeño de nuestras labores de investigación y de enseñanza y para colaborar con otros investigadores y con alumnos. 


\section{Metodología}

El Departamento de Arqueoastronomía necesitaba enriquecer y organizar su acervo bibliográfico para poder desempeñar sus labores con más solvencia. El esfuerzo seria obtener por todos los medios posibles libros, revistas, artículos, videos y sitios web con información sobre arqueoastronomía y ciencias relacionadas.

Se planteó un inventario del material bibliográfico con que disponía el departamento de Arqueoastronomía, para así evaluar las necesidades y fortalezas inmediatas para la formación del Centro de Documentación. Este material se recopiló, se inventarió, se organizó y está disponible para profesores y alumnos.

Se acondicionó un área de trabajo y se colocó la literatura existente previa organización. (Ver figura 1). Se contactó a los particulares e instituciones para lograr donaciones y/o canjes, Se buscó presupuesto para comprar libros o suscripciones de revistas, se elaboraron fichas para todas las referencias, se obtuvo material digital y se colocó en carpetas; información de sitios de internet científicos, videos para presentaciones y se revisaron centros de documentación institucionales.

El material de página web se organizó por carpeta, agrupando la información ya sea por regiones, países, autores, temas o años. En cuanto a material físico (libros, revistas, artículos), se lleva un control para aquellos investigadores que deseen extraerlos de la oficina donde se encuentren. El préstamo para consulta se realiza únicamente dentro de las instalaciones de las FACES. En aras de la conservación de este material, su uso para fotocopias es limitado, a criterio del encargado de este material.

Entre las fuentes de recopilación de material bibliográfico se encontraban:

- Biblioteca Nacional

- Instituto Hondureño de Antropología e Historia

- Hemeroteca Nacional

- Biblioteca Central de la UNAH, entre otras.

El Centro de Documentación cuenta con un encargado dentro del departamento de Arqueoastronomía para el manejo exclusivo del Centro y un asistente para su operación. 

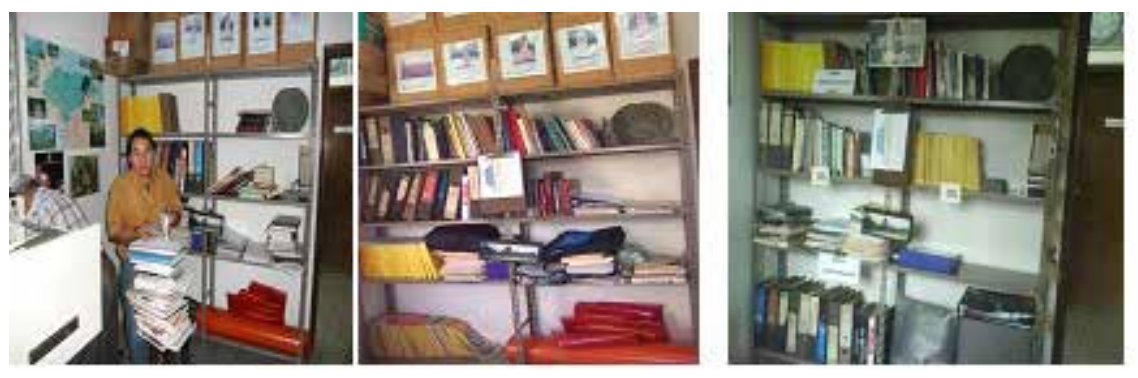

Figura 1. Proceso de organización, inventario y acondicionamiento del Centro de Documentación Arqueoastronómica y Ciencias Afines.

\section{Resultados}

\section{Adecuación de espacio para Centro de Documentación:}

Con la colaboración del Jefe del Departamento de Arqueoastronomía, Vito Veliz, se logró disponer de un espacio adecuado y mobiliario para el inventario preliminar de las obras científicas existentes en los estantes y oficina. Este material fue organizado y clasificado además se realizó un proceso de limpieza y fumigado. Se verificó el estado de los libros, revistas y demás material existente, elaborándose un registro que conformara el comienzo de la base de datos digital del centro de documentación, a ampliarse a medida que se obtengan más ejemplares y acorde al crecimiento del departamento de Arqueoastronomía.

\section{Identificación de necesidades de Departamento:}

Se determinó la necesidad de contar con estantes, computadora para instalación de la base de datos del Centro de documentación, material de oficina (impresoras y tinta, papel bond, lápices y otros). Se detectó la carencia de material bibliográfico específico relacionado con investigaciones arqueoastronómicas y la necesidad de contar con una base de datos de bibliografía digital.

En suma, se ha logrado montar un centro de documentación único en su género, organizado y accesible, para profesores y estudiantes que cuenta con libros, mapas, videos, revistas y sitios web. Además cada ejemplar cuenta con fichas elaboradas que permitirán un mejor manejo. También se logró establecer la organización del material y de la información bibliográfica, permitiendo un centro de 
documentación operable, que posibilita desarrollar convenientemente la docencia e investigación en el departamento de Arqueoastronomía y la Facultad de Ciencias Espaciales.

Se elaboró un listado general de material bibliográfico científico que le servirá de apoyo a los docentes del Departamento de Arqueoastronomía en la impartición de la Asignatura Optativa "Introducción a la Arqueoastronomía". Beneficiando tanto a Profesores como estudiantes y actualizando conocimientos recientes sobre Antropología, Arqueología, Astronomía, arqueoastronomía y las técnicas que se aprovechan en estos campos del saber. $Y$ otras áreas de interés.

\section{Obtención de material bibliográfico (libros, revistas, otros):}

La adquisición de bibliografía para el Centro de Documentación se ha podido conseguir con donaciones o por medio de la compra de volúmenes de material bibliográfico en el mercado nacional e internacional, por medio de la colaboración voluntaria de los docentes del Departamento de Arqueoastronomía y estudiantes, más las donaciones de libros de profesores visitantes (Ver figura 2). También se ha podido recurrir a fotocopias del material bibliográfico ya existente en bibliotecas nacionales, sobre todo las copias antiguas y únicas de gran valor histórico y científico, esto lo permite la Ley de derechos de autor, con fines educativos y no comerciales.

Bertilio Amaya recopilo material de las páginas web científicas el que organizo por carpetas de documentos Pdf, referente a artículos de arqueoastronomía, antropología, arqueología, astronomía y otros. Esto con miras a una colección digital bibliográfica científica de arqueoastronomía y una futura videoteca, para la cual ya se cuentan con numerosos videos científicos.

Muchos documentos se identificaron visitando bibliotecas y centros de investigación especializados, pero su copiado, escaneado o encuadernado resultan onerosos por ahora, por lo que se espera a futuro su obtención por medio asignación de fondos, proyectos o donaciones. 

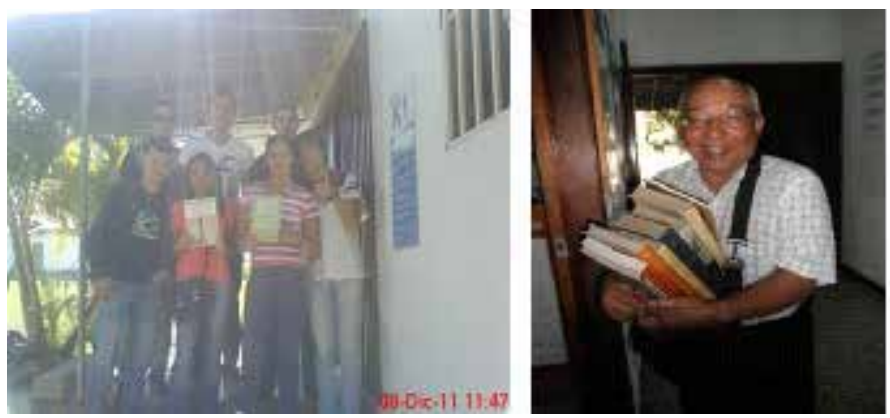

Figura 2. Donaciones de material bibliográfico por medio de la colaboración voluntaria de los docentes del Departamento de Arqueoastronomia y estudiantes.

\section{Organización y equipamiento del Centro:}

La obtención de los fondos para hacer operable del Centro de Documentación, se puede solucionar con donaciones y colaboraciones. Se han realizado las respectivas solicitudes a la jefatura del departamento y decanato para el apoyo a las actividades del centro de documentación con resultados favorables.

Resalta la labor del profesor Vito Veliz que al término de esta investigación ha realizado una importante donación de un nuevo material bibliográfico al centro de documentación Arqueoastronómica, el cual se procederá registrar y organizar a medida que el tiempo lo permita.

Se decidió que el profesor Cesar Rodríguez sería el encargado dentro del departamento de Arqueoastronomía para el manejo del Centro de Documentación y su asistente Bertilio Amaya, con la colaboración de los demás docentes del departamento.

A continuación se detalla la información importante relacionada al funcionamiento del Centro de Documentación y como aprovechar este valioso recurso de investigación científica. 
Cómo está organizado el Centro de Documentación Arqueoastronómica y ciencias Afines:

El Centro de Documentación cuenta con las siguientes áreas temáticas de literatura científica:

- ARQUEOASTRONOMIA MAYA (ver Figura 3).

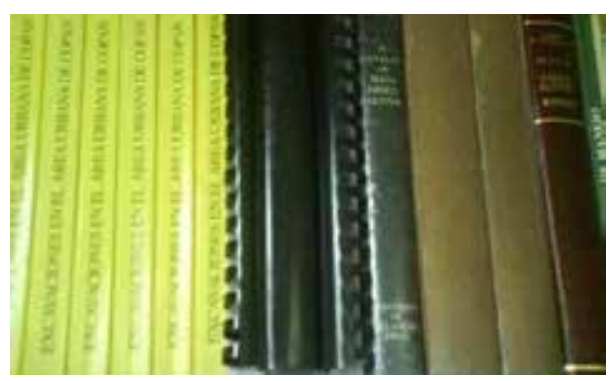

Figura 3. Vista de documentación de la Sección de Arqueoastronomía Maya

- ANTROPOLOGIA

- ARQUEOLOGIA

- ARQUEOASTRONOMIA

- HISTORIA

- OTROS (Otras temáticas, Material digital, mapas) (Ver Figura 4).

- REVISTAS CIENTÍFICAS

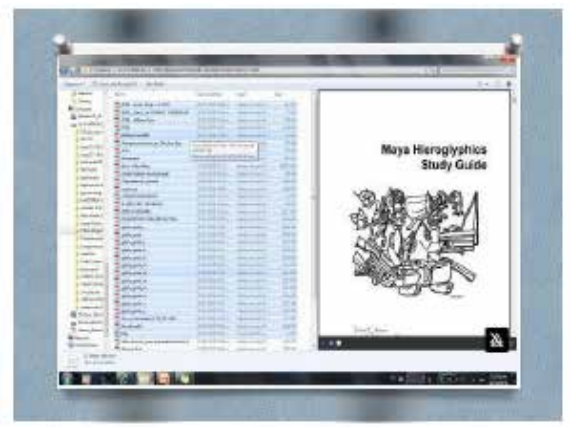

Figura 4. Carpetas de documentos Pdf, referente a artículos de arqueoastronomía, antropología, arqueología, astronomía y otros. 


\section{Cómo se puede hacer uso del Centro de Documentación}

Simplemente apersonándose al Centro de Documentación Arqueoastronómica y Afines ubicado en el edificio K1 de la Facultad de Ciencias Espaciales (FACES) ( Ver Figura 5) y hablar con los encargados Cesar Rodríguez y Bertilio Amaya. A quienes comunicará el título del libro que interesa o el área temática de investigación. Seguidamente se procederá al llenado de la respectiva ficha de uso del libro de interés, el cual se revisará dentro de las instalaciones del centro (préstamo Interno) o por un máximo de tres días, en caso de tener varias copias o no ser material delicado (préstamo externo solo a docentes).

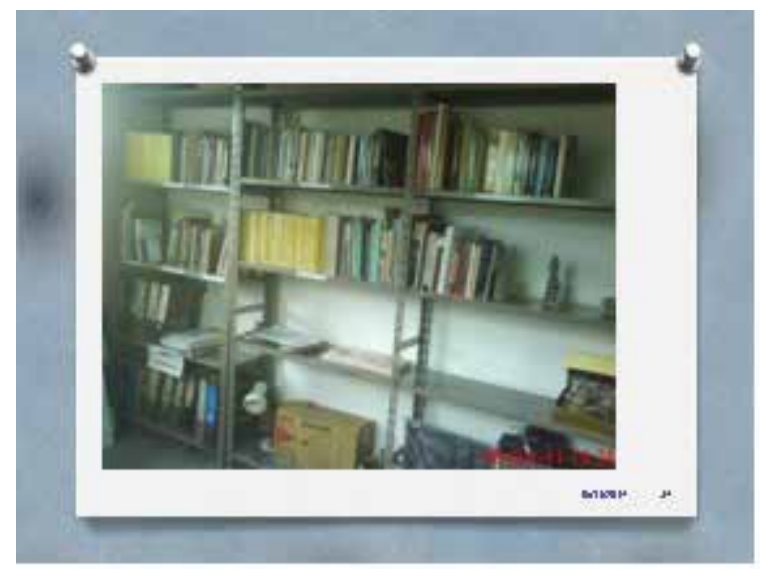

Figura 5. Presentación actual de instalaciones del Centro de Documentación Arqueoastronómica y Ciencias Afines. 


\section{Quiénes pueden usar el Centro de Documentación Arqueoastronómica y Ciencias} Afines

Todo el personal de las FACES, estudiantes y público en general, (Ver Figura 6)

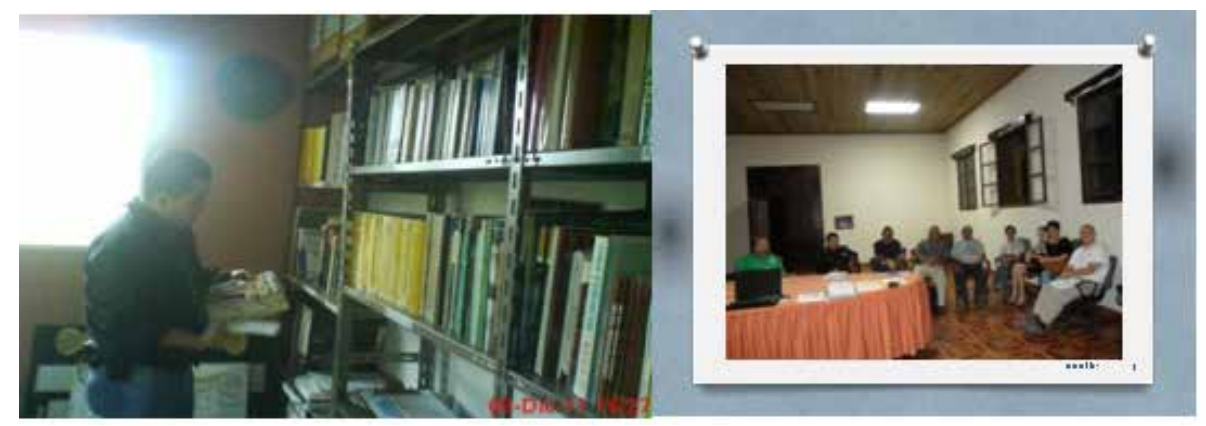

Figura 6. Docente de Arqueoastronomía y alumnos de diplomado haciendo uso del material bibliográfico del Centro de Documentación para sus clases.

Qué horarios está disponible el Centro de Documentación.

Se puede acceder a visitarlo en un horario de 9 am a 4 pm con la salvedad de los horarios de clase de encargados reuniones o compromisos de facultad.

A continuación se presenta la tabla resumen del listado preliminar demostrativo de obras inventariadas en el Centro de Documentación Arqueoastronómica y Ciencias Afines, por el profesor Rodríguez y el instructor Amaya. Se excluyen la mayoría de revistas y otros materiales existentes para uso interno. 
Tabla 1. Listado preliminar resumido con nombre de obra literaria y autor de material bibliográfico disponible en el centro de documentación.

\section{Universidad Nacional Autónoma de Honduras \\ Facultad de Ciencias Espaciales \\ Departamento de Arqueoastronomía y Astronomía Cultural}

\begin{tabular}{|c|c|c|}
\hline \# & Nombre & Autor \\
\hline 1 & $\begin{array}{l}\text { A Catalog of } \\
\text { hieroglyphs }\end{array}$ & Eric S. Thompson \\
\hline 2 & African Sistems of kinship and mariage & $\begin{array}{l}\text { A.R. Radcliffe-Brown, Daryll } \\
\text { Forde }\end{array}$ \\
\hline 3 & America B.C. & Barry Fell \\
\hline 4 & Anales para la Historia de Guatemala & Juan Gavarrete \\
\hline 5 & An Introduction to Anthropology & Beals \& Hoijer \\
\hline 6 & Anthropological Essays & Oscar Lewis \\
\hline 7 & Anales de Antropología (Tomo II) & UNAM \\
\hline 8 & Anales de Antropología (Vol. XV) & UNAM \\
\hline 9 & Anthropology and Ethics & May Edel, Abraham Edel \\
\hline 10 & Archaeology and Volcanism in Central America & Payson Sheets \\
\hline 11 & Archaeology of The Rivas Region, Nicaragua & Paul F. Healy \\
\hline 12 & Atlantis, The Antediluvian world (Dos tomos) & Ignatius Donnelly \\
\hline 13 & Being an Anthropologist, field work in eleven cultures & George D. Spindler \\
\hline 14 & Being a Palauan & H.G. Barnett \\
\hline 15 & Biografía de San Pedro Sula 1536-1954 & Rodolfo Pastor Fasquelle \\
\hline 16 & Book of the Gods and Rites and the Ancient Calendar & Fray Diego Durán \\
\hline 17 & Ceramics and Man & Frederick Matson \\
\hline 18 & Claroscuro del Universo & Mariano Moles Villamate \\
\hline 19 & Códices Mayas & Villacorta y Villacorta \\
\hline 20 & Communication and Social Order & Hugh Dalziel Duncan \\
\hline
\end{tabular}




\begin{tabular}{|l|l|l|}
\hline 21 & Consideraciones sobre la Aritmética Maya & Ruy Díaz Díaz \\
& & \\
\hline 22 & $\begin{array}{l}\text { Convenciones Internacionales y Legislación Panameña sobre la } \\
\text { Defensa del Patrimonio Cultural }\end{array}$ & \\
\hline 23 & $\begin{array}{l}\text { Consulta, Edición y Análisis Espacial con ArcGis 9.2 Tomo I: } \\
\text { Teoria }\end{array}$ & Instituto Nacional de Cultura \\
\hline 24 & Copán y Tikal, Pottery of prehistoric Honduras & John Henderson, Marilyn \\
Beaudry-Corbett
\end{tabular}

\begin{tabular}{|c|c|c|}
\hline & & Central (PRRAC) \\
\hline 30 & Diccionario Español-Maya & Pilar Obón \\
\hline 31 & Didáctica General & \\
\hline 32 & Descubrimiento en el Río Camuy & Russell \& Jeanne Gurnee \\
\hline 33 & Echoes of the Ancient Skies & E.C. Krupp \\
\hline 34 & El Origen de la Vida & Alexandr Ivanovich Oparin \\
\hline 35 & El Imperio Socialista de los Incas & Louis Boudin \\
\hline 36 & El Costo de la Conquista & Linda Newson \\
\hline 37 & Etnologia y Etnohistoria de Honduras & William V. Davidson \\
\hline 38 & Estudios de la Cultura Maya Vol. IX & UNAM \\
\hline 39 & Evolución Histórica de Honduras & Longino Becerra \\
\hline 40 & Evolución Para Todos & Dylan Evans \& Howard Selina \\
\hline 41 & $\begin{array}{l}\text { Excavaciones en el área urbana de Copán } \\
\text { Tomo II: } 2 \text { libros } \\
\text { Tomo III: } 2 \text { libros } \\
\text { Tomo IV: } 10 \text { libros }\end{array}$ & William T. Sanders \\
\hline 42 & Field Projects in Anthropology & Crane Angrosino \\
\hline 43 & Flights Into Yesterday & Leo Devel \\
\hline 44 & Glanz und Untergang des Altem Mexico (Tres tomos) & $\begin{array}{l}\text { Verlag Phillip Von Zabern Mainz } \\
\text { Am Rhein }\end{array}$ \\
\hline 45 & Grandes Misterios de Nuestro Mundo & National Geographic \\
\hline 46 & Hernán Cortés & Grandes Biografias \\
\hline
\end{tabular}




\begin{tabular}{|c|c|c|}
\hline 47 & $\begin{array}{l}\text { Historia de la Astronomía en } \\
\text { México }\end{array}$ & Marco Arturo Moreno Corral \\
\hline 48 & Historia de la Astronomía en México (fotocopia) & Marco Arturo Moreno Corral \\
\hline 49 & Historia Escrita en Piedra & Ricardo Agurcia \& William Fash \\
\hline 50 & Historia de Honduras & Guillermo Varela Osorio \\
\hline 51 & Historia de Olancho 1524-1877 & José A. Sarmiento \\
\hline 52 & $\begin{array}{l}\text { Historia y } \\
\text { de los Mayas }\end{array}$ & Eric S. Thompson \\
\hline 53 & Historia del Puerto de Trujillo & Manuel Rubio Sánchez \\
\hline 54 & Historia de la Universidad & José Reina Valenzuela \\
\hline 55 & Horizons of Anthropology & Sol Tax \\
\hline 56 & Human Heritage, a World History & Greenbaltt Lemmo \\
\hline
\end{tabular}

\begin{tabular}{|l|l|l|}
\hline & & \\
& & \\
& & \\
& & \\
\hline 57 & $\begin{array}{l}\text { Introducción a la Arqueología de Copán, Honduras (Tomos } \\
\text { Iyll) }\end{array}$ & \\
\hline 58 & Incas, Mayas y Aztecas, Mitología & Secretaria de Cultura y Turismo \\
\hline 59 & Indians of North America & Lewis Spence \\
\hline 60 & $\begin{array}{l}\text { Intibucá (1536-1899) Albores del Departamento, Poder } \\
\text { Oligárquico y Pueblos Ancestrales }\end{array}$ & Jesús Evelio Inestroza \\
\hline
\end{tabular}




\begin{tabular}{|c|c|c|}
\hline 61 & La Astronomía en el Antiguo Egipto & José Lull \\
\hline 62 & La Evolución de la Humanidad & E. Perrier \\
\hline 63 & La Guerra en Tierras Mayas & Yvon Lebotl \\
\hline 64 & La Historia antes de la Historia & E. Perrier \\
\hline 65 & La Intendencia de Comayagua & Mario Felipe Martínez Castillo \\
\hline 66 & La Rueda del Tiempo & Carlos Castaneda \\
\hline 67 & La Construcción de Ciudades Vulnerables & Elsa Lily Caballero Zeitún \\
\hline 68 & Las Crónicas perdidas de los Reyes Mayas & David Drew \\
\hline 69 & Lecturas para comprender la Historia de Honduras & Rolando Zelaya y Ferrera \\
\hline 70 & Ley para la Protección del Patrimonio Cultural de la Nación & $\mathrm{IHAH}$ \\
\hline 71 & Los Cakchiqueles en la Conquista de Guatemala & Francis Polo Sifontes \\
\hline 72 & $\begin{array}{l}\text { Man's Rise to Civilization as Shown by the Indians of North } \\
\text { America from Primeval Times to the Industrial State }\end{array}$ & Peter Farb \\
\hline 73 & Maya Archaeologist & Eric S. Thompson \\
\hline 74 & $\begin{array}{l}\text { Memoria VII Seminario de Antropología de Honduras Dr. George } \\
\text { Hasemann }\end{array}$ & $\mathrm{IHAH}$ \\
\hline 75 & Memoria Textual Indígena, elementos de su escritura & Varios autores \\
\hline 76 & Mitos: Alma de Cultura & Vito Véliz \\
\hline 77 & Native Americans Mathematics & Michael P. Closs \\
\hline 78 & Origen africano de la humanidad y los Garínagu precolombinos & Celestino Green \\
\hline 79 & Observadores del Cielo en el México Antiguo & Anthony F. Aveni \\
\hline & 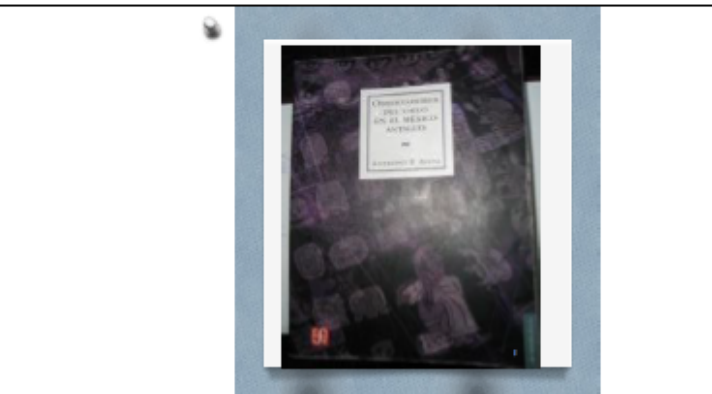 & \\
\hline 80 & Origins of man & Buettner \& Janusch \\
\hline 81 & Plan de manejo zona arqueológica de Copán & IHAH \\
\hline 82 & Popol Vuh & Adrían Recinos \\
\hline 83 & Popol Vuh & Graficentro Editores \\
\hline 84 & Principles of Geomorphology & William D. Thombury \\
\hline 85 & Readings in Anthropology Vol. I & Morton Fried \\
\hline
\end{tabular}




\begin{tabular}{|c|c|c|}
\hline 86 & Reflejo del Cosmos & $\begin{array}{l}\text { Juan Antonio Belmonte \& Michael } \\
\text { Hoskin }\end{array}$ \\
\hline 87 & Relación de las Cosas de Yucatán & Fray Diego de Landa \\
\hline 88 & Remote Sensing in Archaeology & $\begin{array}{l}\text { Stefano Campana \& Maurizio } \\
\text { Forte }\end{array}$ \\
\hline 89 & Rudimentos de Antroplogía & Vito Véliz \\
\hline 90 & San Miguel de Quezailica un Camino Hacia el Reencuentro & Jorge Humberto Orellana \\
\hline 91 & Secretos de dos ciudades Mayas: Copán y Tikal & $\begin{array}{l}\text { Ricardo Agurcia \& Juan Antonio } \\
\text { Valdés }\end{array}$ \\
\hline 92 & Secretos del Pasado & National Geographic \\
\hline 93 & $\begin{array}{l}\text { Ségeda y su Contexto Histórico entre Catón y Nobilior (195-153 } \\
\text { a.C.) }\end{array}$ & Francisco Burillo Mozota \\
\hline 94 & Taller de Arqueoastronomía Maya & $\begin{array}{l}\text { Maria Cristina Pineda de Carias, } \\
\text { Vito Véliz \& M. Martínez }\end{array}$ \\
\hline 95 & $\begin{array}{l}\text { Taller de Capacitación para la enseñanza de la Astronomía en la } \\
\text { Educación Media }\end{array}$ & Rosa M. Ros \\
\hline 96 & $\begin{array}{l}\text { Trabajos de Arqueoastronomía, ejemplos de África, } \\
\text { América, Europa y Oceania }\end{array}$ & José Lull \\
\hline 97 & The Ancient World & Thomas W. Africa \\
\hline 98 & The Ancient World: an Historical Perspective & Henry Boren \\
\hline 99 & The Copán Notes & Varios autores \\
\hline 100 & The Deep Well & Carl Nylander \\
\hline 101 & The Gift & Marcel Mauss \\
\hline 102 & The Native Americans & Spencer \& Jennings \\
\hline 103 & 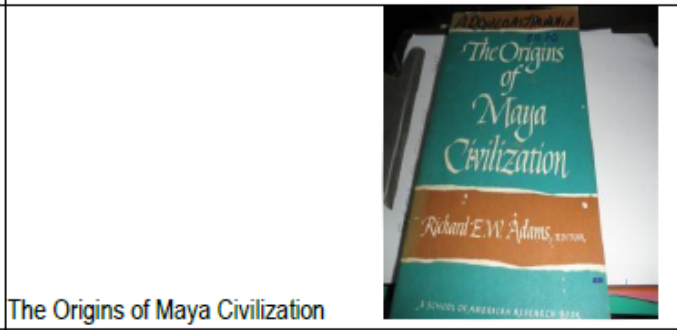 & Richard Adams \\
\hline 104 & The Roots of Ancient India & Walter A. Fairservis Jr. \\
\hline
\end{tabular}




\begin{tabular}{|l|l|l|}
\hline 105 & Trees of Paradise and Pillars of the World & Elizabeth Newsome \\
\hline 106 & & Miguel Tapia Diaz \\
& & \\
& & \\
\hline 107 & Tonalpohualli: Mathesis tolteca & \\
\hline 108 & Visión de los Vencidos & Eric S. Thompson \\
\hline 109 & World Prehistory, an Outline & Miguel León Portilla \\
\hline 110 & $2^{\text {nd }}$ International Conference on Remote Sensing in & Grahame Clark \\
\hline 111 & Archaeology & Stefano Campana \& Mauricio Forte \\
\hline
\end{tabular}

\section{Conclusiones}

Se la logrado obtener libros, revistas, videos y artículos sobre Arqueoastronomía y campos afines, esto por medio de importantes donaciones del jefe de departamento de Arqueoastronomía, docentes y estudiantes de la asignatura optativa Introducción a la Arqueoastronomía, de la Facultad de Ciencias Espaciales.

Se ha logrado encontrar y recopilar sitios Web sobre Arqueoastronomía, con importante información científica disponible en forma de artículos, documentos en Word o Pdf, presentaciones y fotos, además de información de eventos y ultimas noticias.

Con la colaboración de nuestro instructor del departamento de Arqueoastronomía Bertilio Amaya se ha podido organizar todo este acervo bibliográfico para su fácil manejo y uso, y para tener este material a disposición de investigadores y estudiantes, actualizando la información sobre Arqueoastronomía existente, permitiéndonos brindar los mejores y últimos datos científicos, metodologías e información, a los estudiantes, colegas y público en general.

El listado preliminar de material científico literario disponible actualmente en el Centro de Documentación Arqueoastronómica y ciencias Afines, permite afinar la creación de nuestra base de datos bibliográfica, la cual ira enriqueciéndose a medida que se logren más donaciones y obtención de libros, revistas y otros. 


\section{Bibliografía}

- Baudez, Claude F. (1966) Niveaux céramiques au Honduras: une reconsidération de l'évolution culturelle. Journal de la Société des Américanistes. LV, 2:299342. París.

- Chinchilla, Jesus. (1982) Copan Ruins: Complete guide of the great Mayan city. Fifth Edition. Tegucigalpa.

- Complutum. Universidad Complutense de Madrid. Vol. 20. Núm. 2. 2009

- Lull, Jose (2006) Trabajos de Arqueoastronomia. Agrupación Astronómica de la Safor. Valencia.

- Stone, Doris (1957) The archaeology of central and southern Honduras. Papers of the Peabody Museum of Archaeology and Ethnology. Vol. 49, No. 3. Cambridge, Massachusetts.

- Véliz, Vito (1983) Síntesis histórica de la arqueología en Honduras. Yaxkin, Vol. VI, Nos. 1 y 2: 18. Órgano de Divulgación del Instituto Hondureño de Antropología e Historia, Tegucigalpa.

- Véliz, Vito (1988) Rudimentos de Antropología. Litografía López. Tegucigalpa.

\section{Internet:}

- Artículos de Arqueoastronomía. En línea. http://www.arqueoastronomia.org/articulos.htm (Consulta mayo 2011)

- Arqueoastronomia.org. Sitio dedicado a la difusión de la Arqueoastronomía en Hispanoamérica. http://www.arqueoastronomia.org/ (Consulta mayo 2011) 